\title{
Imported malaria in the Infectious Diseases Hospital of laşi: epidemiological and clinical characteristics of 28 consecutive cases
}

\author{
Andrei Vâță ${ }^{*}$, Daniela Leca ${ }^{1}$, Mihaela Cătălina Luca', Denisa Colesniuc², Cătălina Logigan², Denisa Covaciuc², \\ Carmen Dorobăț ${ }^{1}$
}

From The 9th Edition of the Scientific Days of the National Institute for Infectious Diseases Prof Dr Matei Bals

Bucharest, Romania. 23-25 October 2013

\section{Background}

This paper aims to describe the main characteristics of malaria cases observed in the Infectious Diseases Hospital of Iaşi in the last 16 years.

\section{Methods}

We performed a retrospective study of the medical charts of 28 consecutive patients diagnosed with malaria between January 1998 and August 2013. The parameters analyzed were: demographical, related to the stay in the endemic country, clinical, hematological and biochemical parameters, parasitological findings, diagnosis method, treatment and prophylaxis, and days of hospital stay.

\section{Results}

The mean annual number of cases was 1.75 . The patients were aged between 18 and 60 years (mean age 32.1 years). $96.7 \%$ were males. The most frequent country of origin was Turkey (57.1\%), followed by Angola (17.8\%), China (7.1) and India, Ivory Coast, Ethiopia, Portugal, Equatorial Guinea $-3.6 \%$ of cases. The length of stay in the endemic country was in $82.1 \%$ of cases of more than one month. Only $21.4 \%$ of patients had received chemoprophylaxis. Fever higher than $39^{\circ} \mathrm{C}$ was present in all cases. Chills, myalgia, jaundice, vomiting and sometimes neurological symptoms were also present. $25 \%$ were severe forms requiring intensive care. Plasmodium vivax was responsible for $64.3 \%$ of cases, $P$ falciparum $-28.6 \%$, $P$ malariae and $P$ ovale $-3.6 \% .75 \%$ of cases had anemia, $42.8 \%$ thrombocytopenia, $78.6 \%$ elevated ESR, and $28.6 \%$ renal failure. The treatment was started as soon as the diagnosis was established, 4.38 days (mean) after the disease onset. The mean hospitalization time was 11.9 days. $32.1 \%$ of patients had to be transferred to the Clinical Hospital of Infectious and Tropical Diseases "Dr. Victor Babeş", Bucharest, due to disease's severity, lack of medication or response to the initial anti-malarial regimen. No deaths were recorded.

\section{Conclusion}

Although a rare disease in our area, malaria can still pose special diagnostic problems and lead to severe clinical forms in returning travelers from endemic regions.

\section{Authors' details}

1"Gr. T. Popa" University of Medicine and Pharmacy, Iași, Romania. ${ }^{2}$ Infectious Diseases Hospital laşi, Romania.

Published: 16 December 2013

doi:10.1186/1471-2334-13-S1-P83

Cite this article as: Vâță et al:: Imported malaria in the Infectious

Diseases Hospital of laşi: epidemiological and clinical characteristics of 28 consecutive cases. BMC Infectious Diseases 2013 13(Suppl 1):P83.

\footnotetext{
* Correspondence: andreiandrei@yahoo.com

" "Gr. T. Popa" University of Medicine and Pharmacy, Iaşi, Romania
}

Full list of author information is available at the end of the article

(c) 2013 Vâță et al; licensee BioMed Central Ltd. This is an Open Access article distributed under the terms of the Creative Commons Attribution License (http://creativecommons.org/licenses/by/2.0), which permits unrestricted use, distribution, and reproduction in any medium, provided the original work is properly cited. 